\title{
Minimally invasive parathyroidectomy with or without intraoperative parathyroid hormone for primary hyperparathyroidism
}

\author{
Hyun Gu Kim, Woo Young Kim, Sang Uk Woo, Jae Bok Lee, Yu-Mi Lee ${ }^{1}$ \\ Department of Surgery, Korea University Guro Hospital, Korea University College of Medicine, Seoul, 'Department of \\ Anesthesiology and Pain Medicine, Asan Medical Center, University of Ulsan College of Medicine, Seoul, Korea
}

\begin{abstract}
Purpose: The improvement of intraoperative parathyroid hormone (IOPTH) assay and localization studies has enabled a minimally invasive parathyroidectomy (MIP) in primary hyperparathyroidism (pHPT). The aim of this study is to analyze the demographics, clinical presentations, and surgical outcomes of the pHPT patients who received surgical management with versus without IOPTH.

Methods: Analysis of a database was performed on 53 patients who underwent parathyroidectomy for pHPT from 2004 to 2013. Preoperative localization was done by both sestamibi scan and ultrasonography. We divided the patients into two groups (without IOPTH versus with IOPTH) and analyzed the surgical outcomes statistically between two groups.

Results: The concordance rate of Technetium $99 \mathrm{~m}$ sestamibi scan and ultrasonography was $73.6 \%$ and $90.6 \%$, respectively. The overall cure rate of group 1 (without IOPTH) was $94.9 \%$ and that of group 2 (with IOPTH) was $100 \%$. The decline of PTH at postoperative 5 minutes and 10 minutes was $75.2 \% \pm 14.9 \%$ and $84.9 \% \pm 8.6 \%$ in cured patients. On the other hand, that of noncured patients at 5 minutes and 10 minutes was $17.2 \% \pm 9.7 \%$ and $8.2 \% \pm 2.2 \%$. There was a significant difference in the drop rate of IOPTH between cured and persistent patients $(P<0.01)$. Pathological examination showed adenoma in 41 of 53 patients $(77.4 \%)$ and hyperplasia in 10 of 53 patients (18.9\%).

Conclusion: Even though the localization studies were successful, IOPTH monitoring is essential to avoid a surgical failure in MIP.

[Ann Surg Treat Res 2015;89(3):111-116]
\end{abstract}

Key Words: Primary hyperparathyroidism, Parathyroidectomy, Parathyroid hormone, Ultrasonography, Technetium Tc 99m sestamibi

\section{INTRODUCTION}

Primary hyperparathyroidism (pHPT) is a common clinical endocrine disorder in western countries [1]. More recently, the prevalence of PHPT has increased compared to the past owing to development of biochemical tests [2]. Untreated pHPT can cause a cardiovascular disease, which becomes the cause of reduced survival rates [3].
pHPT can only be treated by the parathyroidectomy [4]. The guidelines from the Third International Workshop on the management of asymptomatic pHPT clarified the surgical indication of pHPT patients [5]. pHPT has been treated by traditional bilateral neck exploration (BNE) using intraoperative parathyroid hormone (IOPTH) with a cure rate of more than 95\% [6]. The improvement of IOPTH assays and localization studies have enabled a minimally invasive parathyroidectomy
Received February 5, 2015, Reviewed March 27, 2015,

Accepted April 3, 2015

Corresponding Author: Yu-Mi Lee

Department of Anesthesiology and Pain Medicine, Asan Medical Center, University of Ulsan College of Medicine, 88, Olympic-ro 43-gil, Songpa-gu, Seoul 138-736, Korea

Tel: +82-2-3010-3870, Fax: +82-2-3010-6790

E-mail: ylee@amc.seoul.kr
Copyright (c) 2015, the Korean Surgical Society

(c) Annals of Surgical Treatment and Research is an Open Access Journal. All articles are distributed under the terms of the Creative Commons Attribution NonCommercial License (http://creativecommons.org/licenses/by-nc/4.0/) which permits unrestricted non-commercial use, distribution, and reproduction in any medium, provided the original work is properly cited. 
(MIP) [7]. Many studies reported that the use of IOPTH enables the operators to find and excise additional abnormal parathyroids missed by localization study [8,9]. However, some authors have announced that IOPTH are useless in MIP if pathologic parathyroids are successfully localized $[4,10,11]$.

The aim of this study is to analyze the demographics, clinical presentations and surgical outcomes of the PHPT patients who received surgical management with versus without IOPTH and to investigate the difference of IOPTH drop rate according to time.

\section{METHODS}

Analysis of a database was performed on all patients who underwent parathyroidectomy for PHPT by a single surgeon from 2004 to 2013 at Korea University Guro Hospital. Familial disease and secondary hyperparathyroidism were excluded. Fifty-three patients with sporadic PHPT were eligible for the study.

Serum calcium, serum PTH, 24-hour urine calcium, and bone density were measured at the initial visit. All patients were evaluated with both sestamibi scan and ultrasonography preoperatively for localization. All ultrasonographies and sestamibi scans were reviewed by the surgeon and the radiologist. If pathologic parathyoids were found in sestamibi scan and the results of sonography was concordant with the results of sestamibi scan, patients were offered MIP without IOPTH (group $1, n=39$ ). If any localization studies failed to find the parathyroid or absence of concordance between the results of sestamibi scan and ultrasonography was found, patients underwent the MIP or the BNE using IOPTH (group 2, $\mathrm{n}=14)$.

In group 1, all patients had received MIP, which was performed with a lower neck skin incision, and localized parathyroid was removed. In group 2, patients with enlarged parathyroid in sonography underwent MIP and we checked IOPTH to determine the need for more exploration. But patients in whom localized parathyroid failed to be found in both studies had BNE using IOPTH through a cervical collar incision. The baseline of IOPTH level was measured before parathyroid resection. Also, we measured the IOPTH at 5 and 10 minutes after excision (postoperative 5 minutes, postoperative 10 minutes). IOPTH assays were performed in all groups, but we could not check the results of IOPTH for patients of group 1 because the results of IOPTH were reported postoperatively. The IOPTH level was determined with an Elecsys 2010 apparatus (Roche Diagnostics Co., Indianapolis, IN, USA).

In group 2, the indication of successful operation was the decline of IOPTH over 50\% at any time. Routine frozen biopsy of parathyroids was not carried out. If the intrathyroid nodule was found in preoperative ultrasonography, partial thyroidectomy was combined.

Curative parathyroidectomy was defined as maintaining normocalcemia for at least 6 months after surgery. The criteria of normocalcemia was from 8.5 to $10.2 \mathrm{mg} / \mathrm{dL}$. Persistence of hyperparathyroidism was defined as persistent hypercalcemia within 6 months of the initial operation. Recurrence of hyperparathyroidism was defined as elevated calcium at or beyond 6 months postoperatively. Data were obtained by reviewing hospital records. All patients were followed for at least 6 months. Follow-up information was obtained through patient visits or phone contact.

Stastistical evaluation was performed using IBM SPSS Statistics ver. 20.0 (IBM Co., Armonk, NY, USA). We analyzed the difference of IOPTH drop rate between cured group and noncured group. A P-value less than 0.05 was considered as statistical significance in all analyses.

\section{RESULTS}

Among 53 patients, 34 (64.2\%) were female and 19 (35.8\%) were male. Mean age was $52.8 \pm 15.5$ years. At the time of the first presentation, 35 of the 53 patients $(66.0 \%)$ were asymptomatic. 10 patients (18.9\%) had symptoms related to ureteral stones. Neurological symptoms such as stupor and depressive mood change were revealed in three patients (5.7\%). Four patients $(7.5 \%)$ complained of bone related symptoms; All of them had a history of fracture. One patient who came to the emergency room was diagnosed with acute pancreatitis with hypercalcemia (Table 1).

Table 2 shows the demographic, laboratory, clinical and surgical data of all patients. The mean preoperative serum calcium was $11.6 \pm 1.1 \mathrm{mg} / \mathrm{dL}$, the serum ionized calcium was $5.9 \pm 0.6 \mathrm{mg} / \mathrm{dL}$, and the 24-hour urinary calcium excretion was $254.3 \pm 174.6 \mathrm{mg} /$ day. Patients diagnosed with osteoporosis ( $\mathrm{T}$ score $<-2.5)$ were $24(45.2 \%)$ in the preoperative bone density test. The mean $\mathrm{T}$ score of patients was $-2.5 \pm 1.0$.

The sestamibi scan results of only 39 patients (73.6\%) corresponded with their surgical findings. But, preoperative ultrasonography findings of 48 patients $(90.6 \%)$ showed correlation with operative findings. All 5 patients who failed

Table 1. Symptoms associated with primary hyperparathyroidism

\begin{tabular}{lc}
\hline \multicolumn{1}{c}{ Symptom } & No. of patients $(\%)$ \\
\hline None & $35(66.0)$ \\
Renal and ureteral stone & $10(18.9)$ \\
Neurologic & $3(5.7)$ \\
Bone related & $4(7.5)$ \\
pancreatitis & $1(1.9)$ \\
Total & $53(100)$ \\
\hline
\end{tabular}


Table 2. Demographic, laboratory, clinical and surgical outcomes of primary hyperparathyroid patients

\begin{tabular}{|c|c|}
\hline Variable & Value \\
\hline \multicolumn{2}{|l|}{ Sex } \\
\hline Male & $19(35.8)$ \\
\hline Female & $34(64.2)$ \\
\hline Age (yr) & $52.8 \pm 15.5$ \\
\hline MIBI correlation & $39(73.6)$ \\
\hline Sono correlation & $48(90.6)$ \\
\hline Preop bone density (T score) & $-2.5 \pm 1.0$ \\
\hline Preop 24 hrs urine calcium (mg/day) & $254.3 \pm 174.6$ \\
\hline Preop serum total calcium (mg/dL) & $11.6 \pm 1.1$ \\
\hline Preop serum ionized calcium (mg/dL) & $5.9 \pm 0.6$ \\
\hline Preop basal PTH (pg/mL) & $263.7 \pm 357.3$ \\
\hline Postop PTH (5 min, pg/mL) & $56.9 \pm 52.1$ \\
\hline Postop PTH (10 min, pg/mL) & $35.7 \pm 37.4$ \\
\hline \multicolumn{2}{|l|}{ Neck exploration } \\
\hline Yes & $5(9.4)$ \\
\hline No & $48(90.6)$ \\
\hline \multicolumn{2}{|l|}{ Combined thyroidectomy } \\
\hline Yes & $26(49.1)$ \\
\hline No & $27(50.9)$ \\
\hline \multicolumn{2}{|l|}{ Pathology of parathyroid } \\
\hline Adenoma & $41(77.4)$ \\
\hline Hyperplasia & $10(18.9)$ \\
\hline No pathological glands found & $2(3.8)$ \\
\hline \multicolumn{2}{|l|}{ Pathology of thyroid } \\
\hline Nodular hyperplasia & $14(56.0)$ \\
\hline Papillary thyroid carcinoma & $6(24.0)$ \\
\hline No pathological diagnosis & $5(20.0)$ \\
\hline \multicolumn{2}{|l|}{ Outcome } \\
\hline Cured & $51(96.2)$ \\
\hline Persistence & $2(3.8)$ \\
\hline Recurrence & $0(0)$ \\
\hline
\end{tabular}

Values are presented as number $(\%)$ or mean \pm standard deviation.

MIBI, technetium 99m sestamibi; Sono, ultrasonography; Preop, preoperative; Postop, postoperative; PTH, parathyroid hormone.

localization initially received BNE.

Combined thyroidectomy was performed for 26 patients (49.1\%). Of them, 5 patients (19.2\%) with no preoperative thyroid nodule had a partial thyroidectomy owing to suspicious operative findings, and no pathological diagnosis was confirmed in all. The others had a thyroidectomy due to combined thyroid nodules. Fourteen of 26 patients (56.0\%) had nodular hyperplasia, 6 patients (24.0\%) had papillary thyroid carcinoma, and 5 patients (20.0\%) had no pathological diagnosis finally.

The mean preoperative basal PTH was $228.4 \pm 251.6 \mathrm{pg} / \mathrm{mL}$. The decline of PTH at 5 minutes and 10 minutes postoperative was $75.2 \% \pm 14.9 \%$ and $84.9 \% \pm 8.6 \%$, period of over 6 months in cured patients. On the other hand, that of noncured patients at 5 minutes and 10 minutes was $17.2 \% \pm 9.7 \%$ and $8.2 \% \pm 2.2 \%$,
Table 3. Decline of IOPTH in cured patients and noncured patients

\begin{tabular}{llrl}
\hline & $\begin{array}{c}\text { Cured } \\
(\mathrm{n}=51)\end{array}$ & $\begin{array}{c}\text { Noncured } \\
(\mathrm{n}=2)\end{array}$ & P-value \\
\hline Decline of IOPTH, 5 min $(\%)$ & $75.2 \pm 14.9$ & $17.2 \pm 9.7$ & $<0.01$ \\
Decline of IOPTH, $10 \mathrm{~min}(\%)$ & $84.9 \pm 8.6$ & $8.2 \pm 2.2$ & $<0.01$ \\
\hline
\end{tabular}

Values are presented as mean \pm standard deviation.

IOPTH, intraoperative parathyroid hormone.

respectively. There was a significant difference for the decline of IOPTH between cured and noncured patients (Table 3). There were two patients who were not satisfied with the 50\% drop of IOPTH at 5 minutes after excision. But their IOPTH at 10 minutes decreased below 50\% compared to basal IOPTH and the patients were cured successfully from their pHPT (Fig. 1).

The overall cure rate of 39 patients in group 1 versus 14 patients in group 2 was $94.9 \%$ versus $100 \%$, but there was no stastistical difference. Also, there was no significant differences between the two groups in sex, age, preoperative $\mathrm{T}$ score, 24-hour urine calcium, serum calcium, basal PTH level, postoperative PTH level, combined thyroidectomy or pathology of thyroid and parathyroid (Table 4).

During the follow-up respectively, 51 patients (96.2\%) achieved normocalcemia. There was no recurrence, but persistence of pHPT was shown in 2 patients of group 1. Pathology of excised parathyroids was consistent with adenoma in 41 of 53 patients (77.4\%) and hyperplasia in 10 of 53 patients (18.9\%). There is no complication such as hypocalcemia, hoarseness in all patients.

\section{DISCUSSION}

Since the first parathyroidectomy eighty years ago, the treatment of pHPT had been BNE, which showed excellent results by experienced surgeons [12,13]. Recently, this surgical standard has changed focus to parathyroidectomy due to improvements in preoperative imaging and intraoperative modalities such as IOPTH [14]. Minimally invasive surgeries for $\mathrm{pHPT}$ are associated with short operative time, rapid postoperative recovery and low complication rates $[15,16]$. Especially, many studies have reported that IOPTH monitoring helps to determine the need for complete resection of hyperfunctioning parathyroid in both MIP and BNE [17,18]. In this study, we compared the surgical outcomes in MIP between groups 1 and 2 (without IOPTH versus using IOPTH). All of two persistent cases occured in only group 1 using no IOPTH. There was a significant difference between the two groups regarding surgical failure $(\mathrm{P}<0.01)$.

Measurement of IOPTH in parathyroidectomy was first described by Nussbaum et al. [19]. Compared with baseline PTH level, a decline of $50 \%$ or more in IOPTH means that 

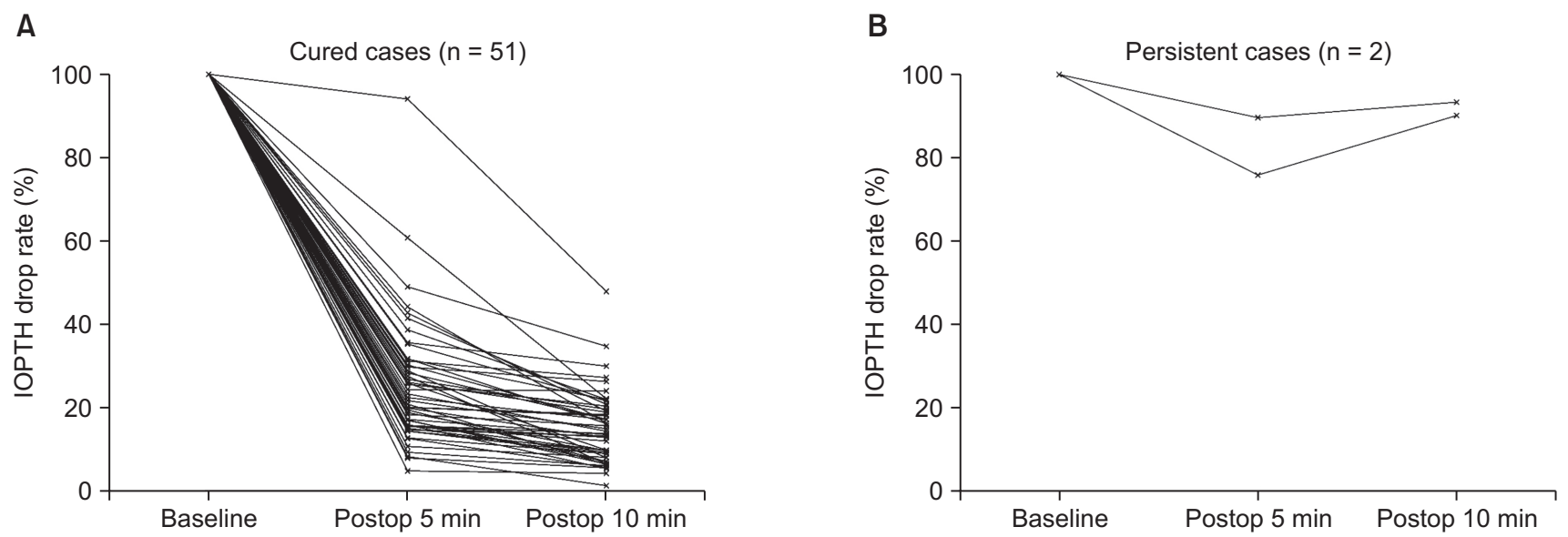

Fig. 1. Results of intraoperative parathyroid hormone in cured patients (A) and in persistent patients (B). IOPTH, intraoperative parathyroid hormone; Postop, postoperative.

Table 4. Comparison of demographic between patients who received the operation without intraoperative PTH (group1) and with intraoperative PTH (group 2)

\begin{tabular}{|c|c|c|}
\hline Variable & Group $1(\mathrm{n}=39)$ & Group $2(n=14)$ \\
\hline \multicolumn{3}{|l|}{ Sex } \\
\hline Male & $13(33.3)$ & $6(42.8)$ \\
\hline Female & $26(66.7)$ & $8(57.2)$ \\
\hline Age $(y r)$ & $52.5 \pm 14.8$ & $53.6 \pm 18.0$ \\
\hline Ultrasonography correlation & $39(100)$ & $9(64.3)$ \\
\hline Preop bone density ( $\mathrm{T}$ score) & $-2.4 \pm 1.0$ & $-2.9 \pm 1.0$ \\
\hline Preop 24-hr u-Ca (mg/day) & $235.7 \pm 128.1$ & $306.1 \pm 264.7$ \\
\hline Preop Ca $(\mathrm{mg} / \mathrm{dL})$ & $11.5 \pm 1.0$ & $11.7 \pm 1.5$ \\
\hline Preop ionized Ca (mg/dL) & $5.9 \pm 0.6$ & $5.8 \pm 0.6$ \\
\hline Preop basal PTH (pg/mL), median (range) & $162.4(34.8-2,076.0)$ & $163.4(59.7-451.8)$ \\
\hline Postop PTH (5 min, pg/mL), median (range) & $37.4(9.9-259.9)$ & $36.3(14.5-152.1)$ \\
\hline Postop PTH (10 min, pg/mL), median (range) & $23.1(5.5-184.3)$ & $23.6(11.2-77.5)$ \\
\hline \multicolumn{3}{|l|}{ Neck exploration } \\
\hline Yes & - & $5(35.7)$ \\
\hline No & $39(100)$ & $9(64.3)$ \\
\hline \multicolumn{3}{|l|}{ Combined thyroidectomy } \\
\hline Yes & $18(46.2)$ & $8(57.1)$ \\
\hline No & $21(53.8)$ & $6(42.9)$ \\
\hline \multicolumn{3}{|l|}{ Pathology of parathyroid } \\
\hline Adenoma & $31(79.5)$ & $10(71.4)$ \\
\hline Hyperplasia & $8(20.5)$ & $2(14.3)$ \\
\hline No pathological glands & - & $2(14.3)$ \\
\hline \multicolumn{3}{|l|}{ Pathology of thyroid } \\
\hline Nodular hyperplasia & $9(50.0)$ & $6(75.0)$ \\
\hline Papillary thyroid carcinoma & $5(27.8)$ & $1(12.5)$ \\
\hline No pathological diagnosis & $4(22.2)$ & $1(12.5)$ \\
\hline \multicolumn{3}{|l|}{ Outcome } \\
\hline Cured & 37 (94.9) & $14(100)$ \\
\hline Persistence & $2(5.1)$ & - \\
\hline Recurrence & - & - \\
\hline
\end{tabular}

Values are presented as number (\%) or mean \pm standard deviation unless otherwise indicated.

u-Ca, urine calcium; Preop, preoperative; Postop, postoperative; PTH, parathyroid hormone. 
hyperfunctioning parathyroid tissue has been excised [20]. There is an argument about the time of PTH measurement after excision [21]. Barczynski et al. [22] had reported that when they analyzed the Halle, Miami, Rome, and Vienna IOPTH Criteria in MIP, each criteria had a distinguishing limitation. Above all, the Miami Criteria, i.e., a decline in IOPTH of $50 \%$ or more at 10 minutes after excision, showned acceptable sensitivity and specificity in detecting multiple gland disease [21]. We checked the IOPTH at 5 and 10 minutes, and two patients had a decline of IOPTH of $50 \%$ or less at 5 minutes. But they finally showed a decline of IOPTH of $50 \%$ or more at 10 minutes. Using the Miami Criteria, we could predict the cure of PHPT in all patients.

Symptomatic pHPT is rare today, but renal and ureteral stone still occurs in $6 \%$ to $15 \%$ of cases [23]. In our study, 10 patients (18.9\%) suffered from nephrolithiasis, which are slightly higher incidence rates than that of other studies. Moreover, symptomatic pHPT in our study was 18 patients (34.0\%), and it is more common than that in the West. The mean age was 52.8 \pm 15.5 years and the ratio of sex was $19: 34$ (male:female). It was not different from previous studies [24].

Identification of pathologic parathyroid is important for deciding on how to operate. Ultrasonography successfully identifies a solitary adenoma in $61 \%-92 \%$ of patients, and when combined with sestamibi has been found to have greater than $90 \%$ sensitivity [25]. Sestamibi scan is a useful modality for finding abnormal glands, and it has been found to be $50 \%$ $91 \%$ sensitive in localization of pathologic glands. But many studies reported variation of sensitivity [26,27]. Several studies had reported that sestamibi scan could not detect a smaller parathyroid due to low resolution [28]. We found that sensitivity of sestamibi scan was only $73.6 \%$, but that of ultrasonography was $90.6 \%$.

Coexistence of thyroid nodules is found in $20 \%-60 \%$ of patients with primary HPT [29]. Those findings should be evaluated preoperatively because there are possibilities for unsuspected thyroid cancer or intrathyroid parathyoid [30]. In our series, combined thyroidectomy was performed in 26 patients (49.1\%). Among them, six patients had a thyroid carcinoma and 15 patients were diagnosed with nodular hyperplasia. But there was no intrathyroid parathyroid.

There are some limitations to our study. First, due to a small number of patients, we could not analyze the variables that influenced persistent or recurrent disease. With regard to this, additional multicenter trials should be carried out. Second, our study has the limitation of a single institution study. It may be subjective to referral bias.

In conclusion, even though the localization studies were successful, IOPTH monitoring is essential to avoid surgical failure in MIP. Especially, the Miami Criteria is a proper tool in evaluation of successful parathyroidectomy.

\section{CONFLICTS OF INTEREST}

No potential conflict of interest relevant to this article was reported.

\section{REFERENCES}

1. Marcocci C, Cetani F. Clinical practice: primary hyperparathyroidism. N Engl J Med 2011;365:2389-97.

2. Lee SW, Park H, Shin JM, Lee YM, Park JH, Koh YW, et al. Clinical analysis of parathyroid adenoma with primary hyperparathyroidism. Korean J OtolaryngolHead Neck Surg 2006;49:72-8.

3. Lundgren E, Lind L, Palmer M, Jakobsson S, Ljunghall S, Rastad J. Increased cardiovascular mortality and normalized serum calcium in patients with mild hypercalcemia followed up for 25 years. Surgery 2001;130:978-85.

4. Ollila DW, Caudle AS, Cance WG, Kim HJ, Cusack JC, Swasey JE, et al. Successful minimally invasive parathyroidectomy for primary hyperparathyroidism without using intraoperative parathyroid hormone assays. Am J Surg 2006;191:52-6.

5. Bilezikian JP, Khan AA, Potts JT Jr; Third International Workshop on the Management of Asymptomatic Primary Hyperthyroidism. Guidelines for the management of asymptomatic primary hyperparathyroidism: summary statement from the third international workshop. J Clin Endocrinol Metab 2009; 94:335-9.

6. Phillips IJ, Kurzawinski TR, Honour JW. Potential pitfalls in intraoperative parathyroid hormone measurements during parathyroid surgery. Ann Clin Biochem 2005:42(Pt 6):453-8.

7. Schneider DF, Mazeh H, Sippel RS, Chen $\mathrm{H}$. Is minimally invasive parathy- roidectomy associated with greater recurrence compared to bilateral exploration? Analysis of more than 1,000 cases. Surgery 2012;152:1008-15.

8. Sebag F, Shen W, Brunaud L, Kebebew E, Duh QY, Clark OH. Intraoperative parathyroid hormone assay and parathyroid reoperations. Surgery 2003;134:104955.

9. Chen H, Mack E, Starling JR. A comprehensive evaluation of perioperative adjuncts during minimally invasive parathyroidectomy: which is most reliable? Ann Surg 2005;242:375-80.

10. Mihai R, Palazzo FF, Gleeson FV, Sadler GP. Minimally invasive parathyroidectomy without intraoperative parathyroid hormone monitoring in patients with 
primary hyperparathyroidism. Br J Surg 2007;94:42-7.

11. Pang T, Stalberg P, Sidhu S, Sywak M, Wilkinson M, Reeve TS, et al. Minimally invasive parathyroidectomy using the lateral focused mini-incision technique without intraoperative parathyroid hormone monitoring. Br J Surg 2007;94:315-9.

12. Augustine MM, Bravo PE, Zeiger MA. Surgical treatment of primary hyperparathyroidism. Endocr Pract 2011;17 Suppl 1:75-82.

13. Low RA, Katz AD. Parathyroidectomy via bilateral cervical exploration: a retrospective review of 866 cases. Head Neck 1998;20:583-7.

14. Jacobson SR, van Heerden JA, Farley DR, Grant CS, Thompson GB, Mullan BP, et al. Focused cervical exploration for primary hyperparathyroidism without intraoperative parathyroid hormone monitoring or use of the gamma probe. World J Surg 2004:28:1127-31.

15. Udelsman R, Lin Z, Donovan P. The superiority of minimally invasive parathyroidectomy based on 1650 consecutive patients with primary hyperparathyroidism. Ann Surg 2011;253:58591.

16. Miccoli P, Bendinelli C, Berti P, Vignali E, Pinchera A, Marcocci C. Video-assisted versus conventional parathyroidectomy in primary hyperparathyroidism: a prospective randomized study. Surgery 1999;126:1117-21.

17. Hughes DT, Miller BS, Doherty GM, Gauger PG. Intraoperative parathyroid hormone monitoring in patients with recognized multiglandular primary hyper- parathyroidism. World J Surg 2011;35:33641.

18. Rajaei MH, Oltmann SC, Adkisson CD, Elfenbein DM, Chen H, Carty SE, et al. Is intraoperative parathyroid hormone monitoring necessary with ipsilateral parathyroid gland visualization during anticipated unilateral exploration for primary hyperparathyroidism: a twoinstitution analysis of more than 2,000 patients. Surgery 2014:156:760-6.

19. Nussbaum SR, Thompson AR, Hutcheson KA, Gaz RD, Wang CA. Intraoperative measurement of parathyroid hormone in the surgical management of hyperparathyroidism. Surgery 1988:104:1121-7.

20. Miura D, Wada N, Arici C, Morita E, Duh QY, Clark OH. Does intraoperative quick parathyroid hormone assay improve the results of parathyroidectomy? World J Surg 2002;26:926-30.

21. Carneiro DM, Solorzano CC, Nader MC, Ramirez M, Irvin GL 3rd. Comparison of intraoperative $\mathrm{PTH}$ assay (QPTH) criteria in guiding parathyroidectomy: which criterion is the most accurate? Surgery 2003;134:973-9.

22. Barczynski M, Konturek A, HubalewskaDydejczyk A, Cichon S, Nowak W. Evaluation of Halle, Miami, Rome, and Vienna intraoperative iPTH assay criteria in guiding minimally invasive parathyroidectomy. Langenbecks Arch Surg 2009:394:843-9.

23. Irvin GL 3rd, Carneiro DM, Solorzano CC. Progress in the operative management of sporadic primary hyperparathyroidism over 34 years. Ann Surg 2004:239:704-8.
24. Khan A, Bilezikian J. Primary hyperparathyroidism: pathophysiology and impact on bone. CMAJ 2000;163:184-7.

25. Adkisson CD, Koonce SL, Heckman MG, Thomas CS, Harris AS, Casler JD. Predictors of accuracy in preoperative parathyroid adenoma localization using ultrasound and Tc-99m-Sestamibi: a 4-quadrant analysis. Am J Otolaryngol 2013:34:508-16.

26. Prasannan S, Davies G, Bochner M, Kollias J, Malycha P. Minimally invasive parathyroidectomy using surgeon-performed ultrasound and sestamibi. ANZ J Surg 2007:77:774-7.

27. Chapuis Y, Fulla Y, Bonnichon P, Tarla E, Abboud B, Pitre J, et al. Values of ultrasonography, sestamibi scintigraphy, and intraoperative measurement of 1-84 PTH for unilateral neck exploration of primary hyperparathyroidism. World J Surg 1996;20:835-9.

28. Chiu B, Sturgeon C, Angelos P. What is the link between nonlocalizing sestamibi scans, multigland disease, and persistent hypercalcemia? A study of 401 consecutive patients undergoing parathyroidectomy. Surgery 2006;140:418-22.

29. Strichartz SD, Giuliano AE. The operative management of coexisting thyroid and parathyroid disease. Arch Surg 1990; 125:1327-31.

30. Morita SY, Somervell H, Umbricht CB, Dackiw AP, Zeiger MA. Evaluation for concomitant thyroid nodules and primary hyperparathyroidism in patients undergoing parathyroidectomy or thyroidectomy. Surgery 2008;144:862-6. 